\title{
HE 0017+0055: A probable pulsating CEMP-rs star and long-period binary ${ }^{\star}$
}

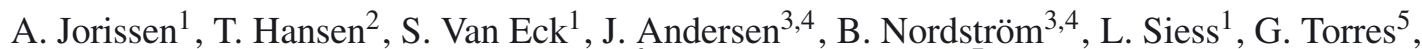 \\ T. Masseron ${ }^{6}$, and H. Van Winckel ${ }^{7}$
}

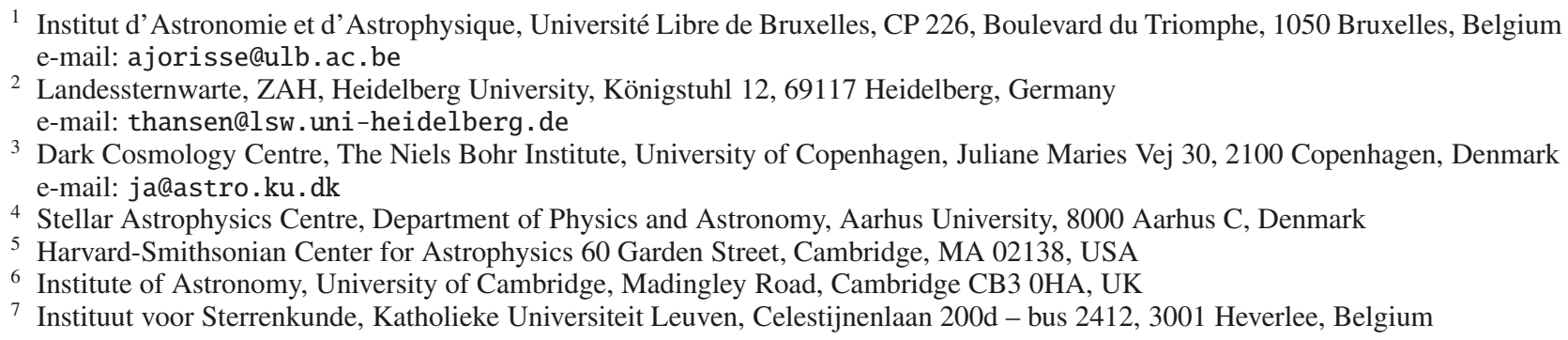

Received 18 July 2015 / Accepted 19 October 2015

\begin{abstract}
Context. A large fraction of the carbon-enhanced, extremely metal-poor halo giants $([\mathrm{Fe} / \mathrm{H}]<-2.5)$ are also strongly enriched in neutron-capture elements from the $s$ process (CEMP-s stars). The conventional explanation for the properties of these stars is mass transfer from a nearby binary companion on the asymptotic giant branch (AGB). This scenario leads to a number of testable predictions in terms of the properties of the putative binary system and the resulting abundance pattern. Among the CEMP stars, some stars further exhibit overabundances in $r$-process elements on top of the $s$-process enrichment, and are tagged CEMP-rs stars. Although the nucleosynthesis process responsible for this kind of mixed abundance pattern is still under debate, CEMP-rs stars seem to belong to binary systems as do CEMP-s stars.

Aims. Our aim is to present and analyse in detail our comprehensive data set of systematic radial-velocity measurements and highresolution spectroscopy of the CEMP star HE 0017+0055.

Methods. Our precise radial-velocity monitoring of HE 0017+0055 over 2940 days (8 yr) with the Nordic Optical Telescope and Mercator telescopes exhibits variability, with a period of $384 \mathrm{~d}$ and amplitude of $540 \pm 27 \mathrm{~m} \mathrm{~s}^{-1}$ superimposed on a nearly linear long-term decline of $\sim 1 \mathrm{~m} \mathrm{~s}^{-1}$ day $^{-1}$. We used high-resolution HERMES/Mercator and Keck/HIRES spectra to derive elemental abundances with 1D LTE MARCS models. A metallicity of $[\mathrm{Fe} / \mathrm{H}] \sim-2.4$ is found, along with $s$-process overabundances of the order of 2 dex (with the exception of [Y/Fe] +0.5), and most notably overabundances of $r$-process elements like Sm, Eu, Dy, and Er in the range $0.9-2.0$ dex. With $[\mathrm{Ba} / \mathrm{Fe}]>1.9 \mathrm{dex}$ and $[\mathrm{Eu} / \mathrm{Fe}]=2.3 \mathrm{dex}, \mathrm{HE} 0017+0055$ is a CEMP-rs star. We used the derived atmospheric parameters and abundances to infer HE 0017+0055 evolutionary status from a comparison with evolutionary tracks.

Results. HE 0017+0055 appears to be a giant star below the tip of the red giant branch. The $s$-process pollution must therefore originate from mass transfer from a companion formerly on the AGB, which is now a carbon-oxygen white dwarf (WD). If the $384 \mathrm{~d}$ velocity variations are attributed to the WD companion, its orbit must be seen almost face-on, with $i \sim 2.3^{\circ}$, because the mass function is very small: $f\left(M_{1}, M_{2}\right)=(6.1 \pm 1.1) \times 10^{-6} M_{\odot}$. Alternatively, the WD orbital motion could be responsible for the long-term velocity variations, with a period of several decades. The $384 \mathrm{~d}$ variations should then be attributed either to a low-mass inner companion (perhaps a brown dwarf, depending on the orbital inclination), or to stellar pulsations. The latter possibility is made likely by the fact that similar low-amplitude velocity variations, with periods close to $1 \mathrm{yr}$, have been reported for other CEMP stars in a companion paper. A definite conclusion about the origin of the $384 \mathrm{~d}$ velocity variations should however await the detection of synchronous low-amplitude photometric variations.
\end{abstract}

Key words. stars: carbon - stars: evolution - stars: individual: HE 0017+0055 - Galaxy: halo

\section{Introduction}

Over the past several years, evidence has been accumulating that as many as $20 \%$ of halo giants with $[\mathrm{Fe} / \mathrm{H}]<-2.5 \mathrm{dex}$ (a proportion rising to $80 \%$ at $[\mathrm{Fe} / \mathrm{H}] \leq-4.0 \mathrm{dex})$ exhibit overabundances of carbon by as much as 1-2 dex (the so-called

$\star$ Based on observations performed with the Mercator telescope and the Nordic Optical Telescope (NOT), operated by the Nordic Optical Telescope Scientific Association at the Roque de los Muchachos Observatory, La Palma, Spain, of the Instituto de Astrofisica de Canarias.
Carbon-Enhanced Metal-Poor stars, CEMP stars; Beers \& Christlieb 2005; Masseron et al. 2010; Placco et al. 2014). Moreover, stars with concomitant enhancement of $s$-process elements (CEMP-s stars) seem to preferentially inhabit the inner halo, while those without $s$-process enhancement (CEMP-no stars) seem to dominate the outer halo (Carollo et al. 2014). The conventional explanation for the origin of the CEMP-s stars, by analogy with barium and $\mathrm{CH}$ stars (McClure \& Woodsworth 1990), is mass transfer from a nearby binary companion on the asymptotic giant branch (AGB) stage that has since evolved into a white dwarf (WD). The mass transfer can occur via Roche-lobe 
overflow or a strong stellar wind, or possibly a combination of both mechanisms (Abate et al. 2013). Indeed, preliminary radial-velocity data by Lucatello et al. (2005) suggested that most or all CEMP-s stars are long-period binaries. The sample then available, however, did not distinguish between CEMP-s and CEMP-no stars, and the results were not conclusive. We have therefore undertaken similar systematic studies to elucidate the origin of these stars in more detail. Simultaneously with the present study, Hansen et al. (2016) addressed the same question, with the conclusion that $80 \%$ of CEMP-s stars are binaries, and CEMP-no stars are generally not. Other than CEMP-no and CEMP-s stars, there is another class of interest in the context of the present paper, namely, the CEMP-rs stars. In addition to large overabundances of elements produced by the $s$ process, they also exhibit large overabundances of elements traditionally related to the $r$ process. The first such stars were discovered by Barbuy et al. (1997) and Hill et al. (2000). A number (if not all) of these stars exhibit radial-velocity variations (e.g. Sivarani et al. 2004; Barbuy et al. 2005; Hansen et al. 2016). The nature of the companion star at the origin of the chemical enrichment of the CEMP star, probably through mass transfer, however, remains debated (Masseron et al. 2010; Hansen et al. 2016). An intermediate-mass AGB star where the ${ }^{22} \mathrm{Ne}(\alpha, n)^{25} \mathrm{Mg}$ neutron source operates is generally invoked.

HE 0017+0055 was included in two independent radialvelocity monitoring programmes, one performed with the HERMES spectrograph attached to the 1.2-m Mercator telescope (Raskin et al. 2011) and addressing many different science cases (Van Winckel et al. 2010); the other with the fibrefed, bench-mounted spectrograph FIES at the Nordic Optical Telescope (NOT); see Hansen et al. (2011). Given the unusual properties of HE $0017+0055$, we decided to combine our data sets and discuss that star separately from the remainder of the samples (see Jorissen et al. 2016, for a discussion of the results of the HERMES monitoring of CEMP-s stars, and Hansen et al. 2015 , for a discussion of the NOT results).

HE $0017+0055$ was discovered in a survey by Stephenson (1985) with a spectrum resembling type R, and received number 39 in the second edition of the General catalog of Galactic carbon stars (GCGCS; Stephenson 1989). It was later rediscovered in the framework of the Hamburg/ESO survey (Christlieb et al. $2001)$ as a high-latitude carbon $\operatorname{star}\left(b=-61^{\circ}\right)$. Based on a fit of selected $\mathrm{Ca}$ and $\mathrm{Fe}$ lines (see Beers et al. 2007b) with synthetic spectra, Kennedy et al. (2011) derived a metallicity of -2.7 for HE $0017+0055$. Hence the star belongs to the family of CEMP stars.

The very low surface gravity $(\log g=0.18)$ adopted by Kennedy et al. (2011) for HE 0017+0055 would make it a very luminous CEMP star, a possible twin of CS 30322-023 (Masseron et al. 2006) and HD 112869 (Začs et al. 2015). Given the scarcity of this kind of low-metallicity AGB stars, finding yet another example appears surprising and warrants the detailed study presented in this paper. In fact, based on the stellar parameters derived in Sects. 3 and 4, our study does not confirm, however, the AGB nature of HE 0017+0055, as we discuss in Sect. 5. We start the paper by presenting the radial-velocity monitoring and the resulting orbital parameters (Sect. 2).

\section{Radial-velocity monitoring and orbital solution \\ 2.1. Radial-velocity monitoring with the HERMES spectrograph}

The HERMES radial-velocity (RV) monitoring was performed with the fibre-fed HERMES spectrograph attached to the $1.2-\mathrm{m}$

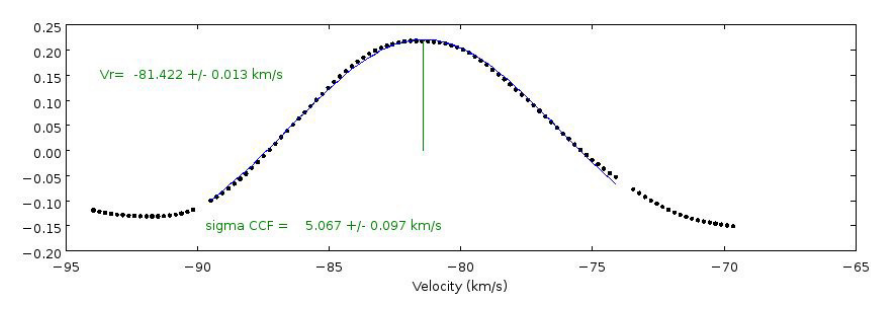

Fig. 1. A typical CCF for HE $0017+0055$ from a spectrum taken with an exposure time of $900 \mathrm{~s}$. The vertical line shows the radial velocity of the star as derived from a Gaussian fit to the CCF core.

Mercator telescope of the Katholieke Universiteit Leuven, installed at the Roque de los Muchachos Observatory (La Palma, Spain) and fully described in Raskin et al. (2011). HERMES is designed to optimise both stability and efficiency and covers the whole wavelength range from 380 to $900 \mathrm{~nm}$ at a resolving power of $\sim 86000$. The high-resolution science fibre has a 2.5 arcsec aperture on the sky, using a two-slice image slicer.

A Python-based pipeline extracts a wavelength-calibrated and a cosmic-ray cleaned spectrum. A separate routine is used for measuring RVs by means of a cross-correlation with a spectral mask constructed from a carbon-star spectrum. A restricted region, covering the range 478.11-653.56 nm (orders 55-74) and containing 2103 useful spectral lines, was used to derive the $\mathrm{RV}$ to avoid telluric lines on the red end and crowded and poorlyexposed spectra on the blue end. A spectrum with a signal-tonoise ratio of 15 is usually sufficient to obtain a cross-correlation function $(\mathrm{CCF})$ with a well-pronounced maximum. A typical CCF is shown in Fig. 1 for a spectrum with a SNR of 15 in the selected spectral region.

Radial velocities are determined from a Gaussian fit to the core of the CCF with an internal precision of a few $\mathrm{m} / \mathrm{s}$. The most important external source of error is the varying atmospheric pressure in the spectrograph room (see Fig. 9 of Raskin et al. 2011), which is largely eliminated by the arc spectra taken for wavelength calibration. The long-term stability (years) of the resulting radial velocities is checked with RV standard stars from Udry et al. (1999). Their standard-deviation distribution peaks at $\sigma\left(V_{\mathrm{r}}\right)=55 \mathrm{~m} / \mathrm{s}$ (see also Fig. 2 of Jorissen et al. 2016), which we adopt as the typical radial-velocity uncertainty for these relatively bright stars. The RV standard stars have also been used to tie the HERMES RVs to the IAU standard system. The HERMES velocities are listed in Table 1.

\subsection{Radial-velocity monitoring at the NOT}

The NOT spectra were obtained in service mode at roughly monthly intervals with the FIES spectrograph ${ }^{1}$, which covers the fixed wavelength range $364 \mathrm{~nm}-736 \mathrm{~nm}$ in 78 orders at a resolving power of $R \sim 46000$. The average SNR of the spectra is $\sim 10$, ranging from $\sim 2$ to $\sim 20$, and is obtained in $\sim 20 \mathrm{~min}$ on a star of $V=14.5$. Integrations of $15 \mathrm{~min}$ or longer are split into three to enable effective cosmic-ray rejection, and a Th-Ar reference spectrum is observed immediately before every stellar spectrum. We observed 2-3 RV standard stars from a fixed set of seven, also selected from Udry et al. (1999), on every observing night to monitor any zero-point variations in the derived velocities.

The observations were reduced and correlated with software developed by Lars Buchhave, originally to deliver high-precision radial velocities of exoplanet host stars from

http://www.not.iac.es/instruments/fies/ 
Table 1. HERMES/Mercator and FIES/NOT radial velocities of HE 0017+0055 (without any zero-point correction).

\begin{tabular}{cccccc}
\hline \hline $\begin{array}{c}\text { JD } \\
(-2400000)\end{array}$ & $\begin{array}{c}V_{\mathrm{r}} \pm \epsilon \\
\left(\mathrm{km} \mathrm{s}^{-1}\right)\end{array}$ & $\begin{array}{c}\mathrm{JD} \\
(-2400000)\end{array}$ & $\begin{array}{c}V_{\mathrm{r}} \pm \epsilon \\
\left(\mathrm{km} \mathrm{s}^{-1}\right)\end{array}$ \\
\hline \multicolumn{6}{c}{ HERMES } \\
\hline 55053.5756 & -79.423 & 0.008 & 54314.6702 & -78.622 & 0.007 \\
55053.5890 & -79.431 & 0.005 & 54338.6419 & -78.944 & 0.007 \\
55053.6025 & -79.382 & 0.009 & 54373.6224 & -78.691 & 0.009 \\
55087.5807 & -79.899 & 0.011 & 54396.5371 & -78.583 & 0.007 \\
55087.5939 & -79.823 & 0.006 & 54406.5966 & -78.505 & 0.012 \\
55087.6072 & -79.851 & 0.006 & 54480.3868 & -78.183 & 0.012 \\
55098.6838 & -79.597 & 0.008 & 54793.4846 & -79.098 & 0.018 \\
55098.6976 & -79.590 & 0.006 & 54820.3386 & -78.830 & 0.017 \\
55098.7124 & -79.738 & 0.007 & 55059.7365 & -79.922 & 0.008 \\
55159.5055 & -79.417 & 0.006 & 55149.4730 & -79.647 & 0.010 \\
55159.5201 & -79.429 & 0.006 & 55207.3499 & -79.391 & 0.015 \\
55159.5343 & -79.463 & 0.014 & 55415.6081 & -80.439 & 0.009 \\
55418.6223 & -80.099 & 0.008 & 55439.5914 & -80.778 & 0.010 \\
55423.7016 & -80.118 & 0.003 & 55503.4086 & -80.330 & 0.023 \\
55497.4806 & -79.992 & 0.007 & 55738.7344 & -80.148 & 0.008 \\
55938.3316 & -79.977 & 0.008 & 55776.6821 & -80.675 & 0.008 \\
55956.3373 & -79.944 & 0.003 & 55821.5766 & -80.870 & 0.009 \\
56109.7113 & -80.056 & 0.005 & 55944.3253 & -80.429 & 0.011 \\
56140.7386 & -80.201 & 0.006 & 56139.7129 & -80.857 & 0.010 \\
56311.3363 & -80.588 & 0.007 & 56241.3910 & -81.420 & 0.018 \\
56481.7027 & -80.278 & 0.007 & 56545.6267 & -81.474 & 0.011 \\
56511.7176 & -80.560 & 0.007 & 56652.4180 & -81.951 & 0.015 \\
56557.6467 & -80.868 & 0.006 & 56686.3209 & -81.538 & 0.015 \\
56634.3763 & -81.416 & 0.007 & 56840.7182 & -80.866 & 0.028 \\
56849.6608 & -80.395 & 0.017 & 56888.5421 & -81.261 & 0.013 \\
56904.6038 & -80.550 & 0.016 & 56917.5981 & -81.398 & 0.014 \\
56947.5245 & -80.919 & 0.014 & 56987.3813 & -81.885 & 0.009 \\
56994.3660 & -81.219 & 0.013 & 57257.5774 & -81.396 & 0.010 \\
56996.3424 & -81.103 & 0.012 & & & \\
57052.3396 & -81.528 & 0.005 & & & \\
57052.3506 & -81.422 & 0.013 & & & \\
57056.3370 & -81.287 & 0.018 & & & \\
57056.3498 & -81.274 & 0.017 & & \\
\hline & & & & & \\
\hline
\end{tabular}

Notes. The uncertainty quoted on the FIES RVs corresponds to the standard deviation of the correlation results from all spectral orders. For the HERMES data, the uncertainty is instead the formal error on the CCF Gaussian fit on 20 orders. A more realistic uncertainty is the long-term error describing the long-term stability of the spectrographs (see text).

echelle spectrographs, especially from FIES (Buchhave et al. 2010). Multi-order cross-correlation and optimised filtering is performed with the highest-signal spectrum of HE 0017+0055 as the template spectrum, and the final radial velocity, given in Table 1, is determined by a Gaussian fit to the peak of the CCF. The internal error of each velocity, also listed, is the standard deviation of the results from all spectral orders. Correlation with a Delta template, i.e. a synthetic spectrum consisting of $\delta$ functions at the (solar) wavelengths of selected lines, is used to determine the radial velocity of the HE $0017+0055$ template and thus that of the whole data set. More details are given in Hansen et al. (2016), from where the NOT data in Table 1 are taken.

The observations of the (much brighter) RV standard stars were reduced exactly as for all science targets. The typical standard deviation is $38 \mathrm{~m} \mathrm{~s}^{-1}$ for a star with $\sim 41$ observations over $\sim 2700$ days, or 7.5 years, and the mean differences between the NOT and Udry et al. (1999) radial velocities is $-13 \pm 38 \mathrm{~m} \mathrm{~s}^{-1}$.

The velocity curve from the combined HERMES+NOT data set covers 2943 days or eight years with a mean radial velocity of $\sim-80 \mathrm{~km} \mathrm{~s}^{-1}$. It is presented in Fig. 2, which clearly shows eight cycles of a short-term periodic velocity
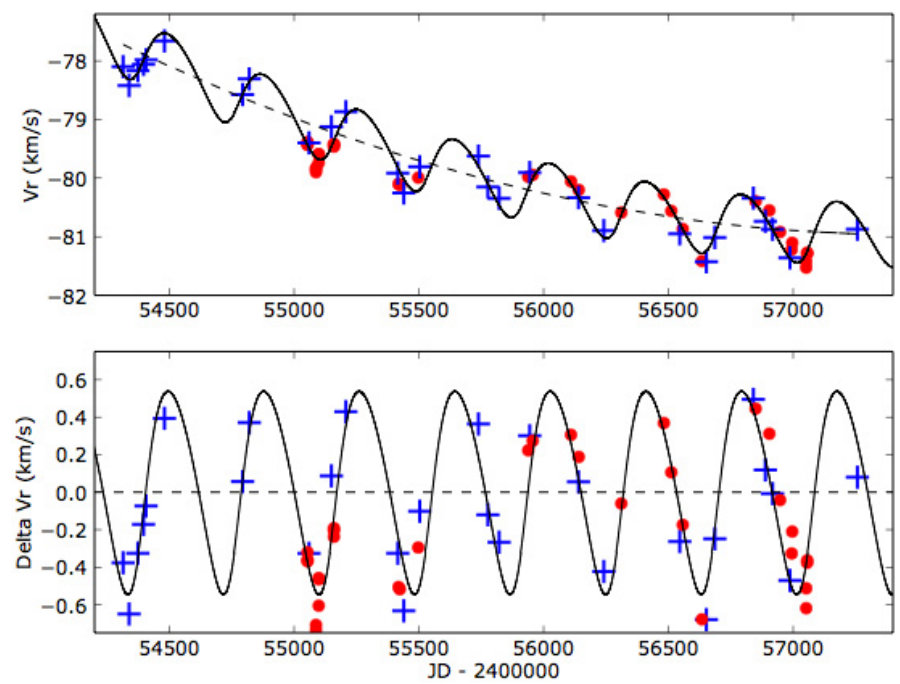

Fig. 2. Radial-velocity curve of HE $0017+0055$ showing the short- and long-term orbital fits combined (top) as well as the short-term orbit alone (red dots: HERMES velocities; blue plusses: NOT velocities).

Table 2. Orbital elements of the presumed short-period pair.

\begin{tabular}{ll}
\hline \hline$P(\mathrm{~d})$ & $384.6 \pm 0.9$ \\
$e$ & $0.15 \pm 0.04$ \\
$\omega\left({ }^{\circ}\right)$ & $234 \pm 16$ \\
$T_{0}$ & $2455908.0 \pm 15.7$ \\
$V_{0}\left(\mathrm{~km} \mathrm{~s}^{-1}\right)$ & $0.04 \pm 0.02$ \\
$K\left(\mathrm{~km} \mathrm{~s}^{-1}\right)$ & $0.54 \pm 0.03$ \\
$f\left(M_{1}, M_{2}\right)\left(M_{\odot}\right)$ & $(6.1 \pm 1.1) \times 10^{-6}$ \\
$a_{1} \sin i(\mathrm{Gm})$ & $2.8 \pm 0.2$ \\
$\sigma\left(\mathrm{km} \mathrm{s}^{-1}\right)$ & 0.14 \\
$N$ & 61 \\
\hline
\end{tabular}

Notes. The table lists the orbital parameters, as follows: $P$ is the orbital period; $e$ is the orbital eccentricity; $\omega$ is the argument of periastron; $T_{0}$ is the time of passage at periastron; $V_{0}$ is the velocity of the barycentre; $K$ is the semi-amplitude of the orbit; $f\left(M_{1}, M_{2}\right)$ is the mass function; $a_{1}$ is the semi-major axis of the orbit of the visible component around the centre of mass of the system; $i$ is the inclination of the orbital plane with respect to the plane of the sky; $\sigma$ is the rms of the O-C residuals; $N$ is the number of data points.

oscillation, superimposed on a long-term trend. We have found that the latter is best modelled by a quadratic polynomial of the form $V_{\mathrm{r}}=3.252 \times 10^{-7} x^{2}-0.0374 x+993.215$, where $x=\mathrm{JD}-2400000$, while the short-term oscillation is adequately represented by a Keplerian orbit.

\subsection{Orbital solution}

From trial orbital solutions of the separate HERMES and NOT, we found that adding a small zero-point correction of $0.52 \mathrm{~km} \mathrm{~s}^{-1}$ to the NOT velocities would minimise the standard deviation of the merged data set in the combined orbital solution, the elements of which are listed in Table 2. Figure 2 shows the velocity curve from the complete HERMES+NOT data set as well as the short-term orbit alone.

With the highly significant radial-velocity amplitude and stable period over eight orbital cycles, and in absence of any photometric evidence for stable pulsations (this aspect is further discussed in Sect. 2.4.3 in relation to Fig. 3), it seems natural to interpret our velocity data as implying that HE $0017+0055$ is a 


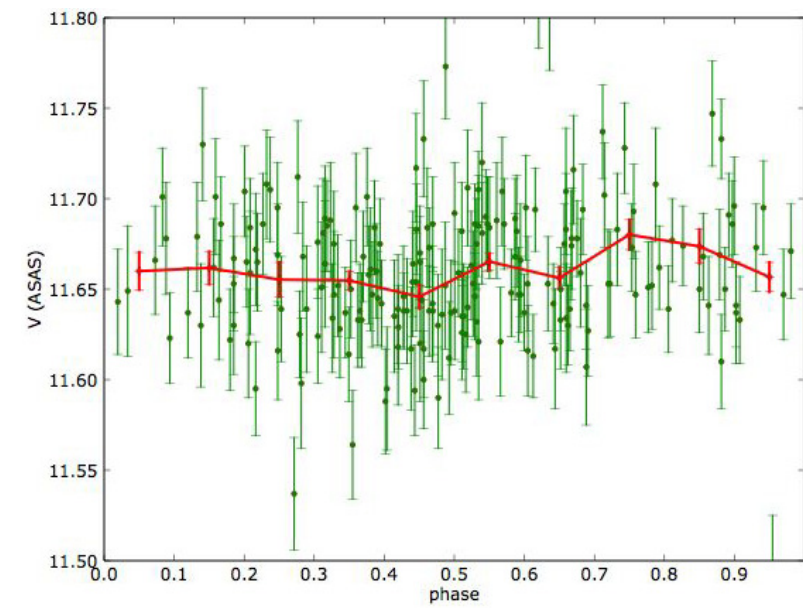

Fig. 3. Photometric data (grade A only) from the ASAS-3 catalogue for HE $0017+0055=$ ASAS $002022+0112.1$, folded on the $383 \mathrm{~d}$ period inferred from the radial-velocity variations. The span of the ordinate axis has been set to eliminate outlying data points. The red curve with error bars corresponds to the average values of the $V$ magnitude in bins of width 0.1 in phase, after eliminating outlying points (i.e. those outside the graphical window). The error bars along that curve correspond to the error on the mean, i.e. the standard dispersion in the considered bin divided by $\sqrt{N}$, where $N$ is the number of data points in the bin.

triple star system with an inner orbit of low eccentricity (which is either seen nearly face-on or with a low-mass companion) and a more distant companion. These alternatives are discussed in the next section (Sect. 2.4). The shorter period is sufficiently different from $365 \mathrm{~d}$ that phases have shifted by about half a cycle over the eight-year span of our monitoring, relative to any residual seasonal effects, and we have documented elsewhere (Hansen et al. 2011) that we can reliably measure binary motions of similar period and even lower amplitude. The outer orbit must have a period of several decades, since we only see marginal evidence of a gradual acceleration over $\sim 3000 \mathrm{~d}$.

Considering the fact that HE 0017+0055 is a CEMP-rs star (as we show in Sect. 4), not residing on the AGB (see Sect. 5 and Fig. 8), it must owe its peculiar abundances to mass transfer in a binary system, such as barium and $\mathrm{CH}$ stars (McClure \& Woodsworth 1990; Gorlova et al. 2013; Jorissen et al. 2016). The most recent collection of orbital elements for these families, presented in the two recent references above, indicates that their orbital periods range between $80 \mathrm{~d}$ and up to $50 \mathrm{yr}$ or more. Both the inner and outer companions of HE 0017+0055 have periods in this range, consistent with that of barium and $\mathrm{CH}$ systems. This argument based on orbital periods cannot be used thus to tag which, among the inner or outer companion, is the WD responsible for the chemical pollution. In case the short-period variations turn out not to be of Keplerian origin (as we argue in Sect. 2.4.3), the long-term velocity variations must then be attributed to the WD companion. As discussed above, this poses no problem in terms of accretion efficiency, since orbital periods of several decades are known among $\mathrm{CH}$ and barium stars.

\subsection{Origin of the short-term velocity variations}

The remarkable feature of the short-term orbit presented in Table 2 is the very small value of its mass function $[(6.1 \pm 1.1) \times$ $\left.10^{-6} M_{\odot}\right]$. In this section, we discuss three possible interpretations of this fact: (i) an orbit with a very low inclination; (ii) a companion of very low mass; and (iii) a spurious Keplerian solution, the velocity variations being caused by envelope pulsations.

\subsubsection{An orbit with a very low inclination?}

The small mass function derived from the orbital fit could imply a very low orbital inclination. Assuming $M_{1}=0.9 M_{\odot}$ for the primary component of $\mathrm{HE} 0017+0055, i \sim 2.3^{\circ}$ is required to yield a secondary mass of $M_{2}=0.6 M_{\odot}$, which is typical for a CO WD. In this situation, it is thus the inner companion that is responsible for the chemical pollution, whereas the outer companion is an innocent bystander. This situation immediately implies that the two orbits cannot be coplanar. If they were coplanar, the semi-amplitude of the outer orbit (assumed to be $3 \mathrm{~km} \mathrm{~s}^{-1}$; Fig. 2), the inferred inclination $\left(2.3^{\circ}\right)$ and period $(\sim 4 \times 8=32$ yr $)$ would imply a mass of several $10^{3} M_{\odot}$ for the outer companion, which is of course impossible. Indeed, many non-coplanar triple systems are known (Hummel et al. 2003; Muterspaugh et al. 2008; O'Brien et al. 2011), and modern theories of stellar formation (Sterzik \& Tokovinin 2002; Fabrycky \& Tremaine 2007) predict that triple systems form non-coplanarily.

\subsubsection{An orbit with a very low-mass companion?}

An alternative solution is that of a very low-mass companion. If $M_{1}=0.9 M_{\odot}$ as assumed above, then the derived mass function imposes that the companion be more massive than $0.017 M_{\odot}$. A brown-dwarf companion is expected for orbital inclinations in the range $12.4^{\circ} \leq i \leq 90^{\circ}, i=12.4^{\circ}$ corresponding to the browndwarf threshold at $M_{2}=0.08 M_{\odot}$. This brown-dwarf companion, if confirmed, would be the most metal poor $([\mathrm{Fe} / \mathrm{H}] \sim-2.4$ as shown in Sect. 3.3) ever discovered, since so far brown-dwarf metallicities are all above $[\mathrm{Fe} / \mathrm{H}] \sim-0.6$ (Burningham et al. 2014 , and references therein), with one possible exception (with a metallicity somewhere between -1 and -2 ; Burgasser et al. 2003).

\subsubsection{Envelope pulsation causing spurious Keplerian-like velocity variations?}

Although the Keplerian interpretations presented above are in principle perfectly acceptable, the study of low-metallicity giants by Carney et al. (2003) and by Jorissen et al. (2016), in a companion paper, invites us to be cautious, however.

Among 13 CEMP-(r)s and CH binary systems, Jorissen et al. (2016) have identified two more cases similar to HE 0017+0055, namely HE 1120-2122 and HD 76396, where small-amplitude variations ( $K$ ranging from 0.1 to $0.9 \mathrm{~km} \mathrm{~s}^{-1}$ ) with periods very close to $1 \mathrm{yr}$ are superimposed on a long-period Keplerian orbit. The CEMP-s star CS 30322-023 also shows a velocity curve similar to that of $\mathrm{HE} 0017+0055$, with short-term radialvelocity variations with a period of $192 \mathrm{~d}$, and a semi-amplitude of $1.7 \mathrm{~km} \mathrm{~s}^{-1}$ that is possibly superimposed on a long-term trend (see Fig. 7 of Masseron et al. 2006).

On the other hand, Carney et al. (2003) reviewed previous evidence that low-metallicity giant stars, especially in globular clusters, systematically exhibit radial-velocity jitter for luminosities near or above the red giant branch (RGB) tip (i.e. $M_{V} \leq-1.5$ or $\log g \leq 1.3$ ). A closer look at these velocity variations reveals that they mimic Keplerian variations, with semi-amplitudes of the order of $1-1.5 \mathrm{~km} \mathrm{~s}^{-1}$ and periods of the order of 170-190 d. Among the cases reported above for CEMP-(r)s/CH systems, only CS 30322-023 conforms to those properties, and the other systems are of lower amplitude and longer period. It is likely, however, that those were out of reach with the spectrograph used by Carney et al. (2003). 
An important property of the velocity variations reported by Carney et al. (2003) is that they are correlated with lowamplitude photometric variations: HIPPARCOS observations of HD 3008 and HD 110281 in Carney's sample, for example, revealed these objects to be variables with (peak-to-peak) amplitudes $A=0.134 \pm 0.045 \mathrm{mag}$ and $A=0.195 \pm 0.032 \mathrm{mag}$, respectively. Most importantly, the photometric and radial-velocity data appear to vary with the same period.

Photometric data for HE 0017+0055 are available from the All Sky Automated Survey (ASAS; Pojmanski 1997), and are shown in Fig. 3, folded on the radial-velocity period of $384 \mathrm{~d}$. No significant modulation has been detected at a $3 \sigma$ level of $0.03 \mathrm{mag}$, where $\sigma=0.01 \mathrm{mag}$ is the typical error bar on the light curve in Fig. 3. Thus, one may exclude (sinusoidal) variations with a peak-to-peak amplitude larger than $0.03 \times 2 \sqrt{2}=$ 0.08 mag in HE 0017+0055.

Should the radial-velocity variations be due to stellar oscillations, they would not contradict the absence of light-variability detection. Indeed, from a simple linear theory of oscillations, Kjeldsen \& Bedding (1995) relate the standard deviation $\sigma_{\lambda}$ (in millimag) of the light variations at wavelength $\lambda$ (in $\mathrm{nm}$ ) to the radial-velocity standard deviation $\sigma\left(V_{\mathrm{r}}\right)$ (in $\mathrm{km} \mathrm{s}^{-1}$ ) caused by adiabatic acoustic oscillations. They find the relation:

$$
\frac{\sigma_{\lambda}}{\sigma\left(V_{\mathrm{r}}\right)}=\frac{21.8}{(\lambda / 550)\left(T_{\mathrm{eff}} / 5777\right)^{2}}
$$

Considering the $0.6 \mathrm{~km} \mathrm{~s}^{-1}$ semi-amplitude $K$ of the velocity variations, which turns into $\sigma\left(V_{\mathrm{r}}\right)=K / \sqrt{2}=0.42 \mathrm{~km} \mathrm{~s}^{-1}$, the above relation predicts $\sigma_{V}=18$ millimag, which is smaller than the detection threshold for the ASAS data estimated above. Thus, the hypothesis that the short-term velocity variations are due to stellar oscillations is consistent with the absence of large light variations in the ASAS light curve.

The expected level of photometric variability in giant stars just below the tip of the RGB, where HE 0017+0055 seems to be located (Sect. 5), has been investigated as well by Kiss \& Bedding (2003) for the Large Magellanic Cloud. These authors conclude that most of these stars are variable, but with variability (peak-to-peak) amplitudes generally smaller than 0.14 mag. The ASAS-3 survey puts an upper limit on the (peak-to-peak) variability amplitude of HE $0017+0055$ at 0.08 mag.

In the absence of more accurate photometric data for HE $0017+0055$, we cannot be sure that the short-term radialvelocity variations are associated with some kind of envelope pulsations. However, the accumulation of systems among CEMP-(r)s/CH stars with low-amplitude velocity variations on a timescale of 190 to $380 \mathrm{~d}$ is puzzling and invites us to be cautious, but at this stage we should not exclude any of the three possibilities described in this and the previous sections.

\section{Stellar parameters}

We now turn to a description of the atmospheric parameters of HE $0017+0055$, necessary to perform the abundance analysis that will reveal the CEMP-rs nature of HE 0017+0055. First, we present a description of its kinematical properties because these properties guide the derivation of the star's surface gravity.

\subsection{Kinematics}

The Tycho-2 project (Høg et al. 2000) derived the propermotion values $\mu_{\alpha} \cos \delta=6.9 \pm 2.7$ mas $\mathrm{yr}^{-1}$ and $\mu_{\delta}=$ $-14.2 \pm 2.5$ mas $\mathrm{yr}^{-1}$. These proper motions (along with the radial velocity of $-80 \mathrm{~km} \mathrm{~s}^{-1}$; see Table 1) have been converted into space velocities $U, V, W$, as this is the basis for an important argument in the choice of the atmospheric parameters. The velocities $U, V, W$ are the heliocentric velocity components along the Galactic coordinate system ( $U$ is positive in the direction of the Galactic centre, and $V$ is positive in the direction of Galactic rotation $l=+90^{\circ}$ ). Adopting different possible locations for HE $0017+0055$ in the Hertzsprung-Russell diagram (on the AGB, on the giant branch, or on the main sequence, along the evolutionary track of a $0.9 M_{\odot}$ star of metallicity $[\mathrm{Fe} / \mathrm{H}]=-2.4$; Table 3), the corresponding luminosities yield distances that are used to convert the Tycho-2 proper motions into components of the space velocity. In this way, we link the atmospheric gravity $\log g$ with the space velocity.

The results are listed in Table 3 and reveal that for gravities lower than $\log g=0.5$, the space velocity is uncomfortably large, since it is likely to exceed the Galactic escape velocity ${ }^{2}$. In the solar neighbourhood, the Galactic escape velocity is estimated to be about 500-600 $\mathrm{km} \mathrm{s}^{-1}$ (Smith et al. 2007), but it must be smaller in the halo at the distance of HE $0017+0055$. We show that the star is unlikely to be a main-sequence star; hence, it must be located at a large Galactic scale height. The $U, V, W$ velocities obtained for $\log g=1$ (Table 3 ) are consistent with a typical halo population, since $V \sim-350 \mathrm{~km} \mathrm{~s}^{-1}$ combined with its estimated metallicity $[\mathrm{Fe} / \mathrm{H}]=-2.4$ (see below) locates HE $0017+0055$ right along the trend observed by Carney et al. (1996) for halo stars (see their Figs. 1, 3, 5, and 8).

\subsection{Atmospheric parameters: previous studies}

Kennedy et al. (2011) derived atmospheric parameters for HE $0017+0055$. The effective temperature $T_{\text {eff }}=4185 \mathrm{~K}$ was estimated from the ( $\left.T_{\text {eff }}, V-K\right)$ relationship of Alonso et al. (1996), with $V-K=2.96$. The $K$ magnitude is from the 2MASS catalogue $(J=9.31 \pm 0.03, H=8.70 \pm 0.03, K=8.50 \pm 0.02$; Cutri et al. 2003), and $V=11.459 \pm 0.008$ and $B=12.991 \pm 0.013$, from Beers et al. (2007a), yielding $B-V=1.532$.

Kennedy et al. (2011) estimated the surface gravity to be $\log g=0.18$ because that value matches $T_{\text {eff }}=4185 \mathrm{~K}$ on the Padova evolutionary tracks for the inferred metallicity of $[\mathrm{Fe} / \mathrm{H}]=-2.5$ (Girardi et al. 2000; Marigo et al. 2001), thus assuming that the star lies on the giant branch and neglecting the possibility that it could be a dwarf carbon star. Uncertainties in $T_{\text {eff }}$ and $\log g$ are $100 \mathrm{~K}$ and 0.5 dex, respectively. The microturbulence is taken to be $2 \mathrm{~km} \mathrm{~s}^{-1}$, consistent with previously determined microturbulence values for giant CEMP stars (Johnson et al. 2007; Aoki et al. 2007). We showed in Sect. 3.1 that a low gravity yields an unrealistic value for the space motion of HE $0017+0055$ (since the inferred high luminosity puts the star at a distance of $14 \mathrm{kpc}$; Table 3), and warrants a revised analysis of the atmospheric parameters as presented in Sect. 3.3.

Nevertheless, adopting the above atmospheric parameters, Kennedy et al. (2011) estimated a metallicity $[\mathrm{Fe} / \mathrm{H}]=-2.72$ for $\mathrm{HE} 0017+0055$, based on a fit of selected $\mathrm{Ca}$ and $\mathrm{Fe}$ lines with synthetic spectra (see Beers et al. 2007b). Kennedy et al. (2011) also derived $[\mathrm{C} / \mathrm{H}]=-0.41,[\mathrm{C} / \mathrm{Fe}]=2.31$, $[\mathrm{N} / \mathrm{Fe}]=0.52,[\mathrm{O} / \mathrm{H}]=-1.7$, and $[\mathrm{O} / \mathrm{Fe}]=1.0$, corresponding to $\log \epsilon(\mathrm{C})=8.02, \log \epsilon(\mathrm{N})=5.64, \log \epsilon(\mathrm{O})=6.99$, adopting $\log \epsilon_{\odot}(\mathrm{C})=8.43, \log \epsilon_{\odot}(\mathrm{N})=7.83$, and $\log \epsilon_{\odot}(\mathrm{O})=8.69$

2 See Pereira et al. (2012) for a case study of high-velocity $\mathrm{CH}$ stars (HD 5223 and BD $-32^{\circ} 1346$ ), near or above the Galactic escape velocity. 
Table 3. Kinematical properties of HE $0017+0055$, for different hypotheses about its absolute visual magnitude $M_{V}$ and, hence, distance, adopting the Tycho-2 proper motion and a $V$ magnitude of 11.65 (Høg et al. 2000, in agreement with the ASAS-3 data; see Fig. 3).

\begin{tabular}{|c|c|c|c|c|c|c|c|c|c|c|c|c|}
\hline $\begin{array}{l}T_{\text {eff }} \\
(\mathrm{K})\end{array}$ & $\log g$ & $\begin{array}{c}L \\
\left(L_{\odot}\right)\end{array}$ & $M_{V}$ & $\begin{array}{c}d \\
(\mathrm{kpc})\end{array}$ & $\begin{array}{c}|z| \\
(\mathrm{kpc})\end{array}$ & $\begin{array}{c}V_{\alpha *} \\
\left(\mathrm{~km} \mathrm{~s}^{-1}\right)\end{array}$ & $\begin{array}{c}V_{\delta} \\
\left(\mathrm{km} \mathrm{s}^{-1}\right)\end{array}$ & $\begin{array}{c}U \\
\left(\mathrm{~km} \mathrm{~s}^{-1}\right)\end{array}$ & $\begin{array}{c}V \\
\left(\mathrm{~km} \mathrm{~s}^{-1}\right)\end{array}$ & $\begin{array}{c}W \\
\left(\mathrm{~km} \mathrm{~s}^{-1}\right)\end{array}$ & Lum. class & Model \\
\hline 4185 & 0.18 & 4470 & -4.1 & 14 & 12.2 & $458 \pm 179$ & $942 \pm 166$ & 68 & -956 & -433 & II & PADOVA \\
\hline 3950 & 0.5 & 1920 & -3.2 & 9.3 & 8.1 & $305 \pm 119$ & $628 \pm 111$ & 49 & -647 & -264 & II & STAREVOL \\
\hline 4000 & 1.0 & 523 & -1.8 & 4.8 & 4.2 & $157 \pm 69$ & $296 \pm 64$ & 29 & -348 & -110 & III & STAREVOL \\
\hline 3950 & 4.9 & 0.03 & 8.8 & 0.037 & 0.032 & $1.2 \pm 0.5$ & $0.15 \pm 0.03$ & 12 & -40 & 68 & $\mathrm{~V}$ & STAREVOL \\
\hline
\end{tabular}

Notes. The uncertainties on the space motion originate from the uncertainties on the Tycho-2 proper motion. The correspondence between $M_{V}$ and $\log g$ is from the STAREVOL track (see Sect. 5) for a $0.9 M_{\odot}$ star with $[\mathrm{Fe} / \mathrm{H}]=-2.4,[\mathrm{C} / \mathrm{H}]=-0.7$, and $\mathrm{C} / \mathrm{O}=20$, adopting a bolometric correction of 0.3 . The first line corresponds, however, to the Padova track used by Kennedy et al. (2011). $U, V, W$ are the heliocentric velocity components along the Galactic coordinate system $(U$ is positive in the direction of the Galactic centre, and $V$ is positive in the direction of Galactic rotation $\left.l=+90^{\circ}\right) . V_{\alpha *}$ and $V_{\delta}$ are the space velocities along the direction of right ascension and declination, respectively.

for the Sun (Asplund et al. 2009). In this paper, we use the standard notation $\log \epsilon(\mathrm{X})=\log [n(\mathrm{X}) / n(\mathrm{H})]+12$, where $n(\mathrm{X})$ is the number density of element $\mathrm{X}$. The CNO abundances of HE 0017+0055 appear normal among CEMP stars (see e.g. Fig. 18 of Masseron et al. 2010). Adopting the solar CNO abundances as listed above, corresponding to $(\mathrm{C} / \mathrm{O})_{\odot}=0.55$, one thus finds $\mathrm{C} / \mathrm{O}=10.7$ for $\mathrm{HE} 0017+0055$. Moreover, Goswami (2005) finds a very low ${ }^{12} \mathrm{C} /{ }^{13} \mathrm{C}$ ratio of 1.3 , typical of (or even lower than) the $\mathrm{CN}$-cycle equilibrium value. This value is the lowest among those reported by both Goswami (2005) and Masseron et al. (2010). Nothing is known about the $s$-process content of HE 0017+0055; this issue therefore is investigated in Sect. 4 as well.

\subsection{Atmospheric parameters revisited}

We rederived the atmospheric parameters of HE $0017+0055$ for the reasons given in Sect. 3.2. We first tried to use the relation between $(V-K),(H-K),(J-K)$ and $T_{\text {eff }}$ derived by Bergeat et al. (2001) for carbon stars to estimate the effective temperature. Unfortunately, the photometric indices for HE 0017+0055 $(V-K=2.96 \pm 0.03, H-K=0.20 \pm 0.05$, $J-K=0.81 \pm 0.05)$ require us to severely extrapolate the calibrations of Bergeat et al. (2001), which were aimed at cool carbon stars of type N. Taken at face value, these calibrations yield temperatures of 4680, 3470, and $3890 \mathrm{~K}$, respectively, and are thus of little use. However, HE 0017+0055 has colours identical to those of the warm R stars studied by Zamora et al. (2009; see their Fig. 1). In that sample, HIP 87603 and HIP 113150 share the same $H-K$ and $J-K$ indices as HE $0017+0055$, and have effective temperatures of 4100 and $4500 \mathrm{~K}$, respectively (and both with $\log g=2.0$ ).

The photometric similarity between HE $0017+0055$ and both $\mathrm{R}$ and $\mathrm{CH} \operatorname{stars}^{3}$ (Zamora et al. 2009) led us to focus our search around $T_{\text {eff }} \sim 4100-4500 \mathrm{~K}$ and $\log g=2.0$. The final atmospheric parameters were derived with an iterative process of fitting specific spectral regions and fine-tuning the parameters consecutively, modifying $T_{\text {eff }}$, gravity, metallicity, $s$-process abundances as a whole, and the CNO abundances until a reasonable fit to the observed spectrum was found, for the whole range $420-800 \mathrm{~nm}$. The synthetic spectra were produced by the TURBOSPECTRUM code (Alvarez \& Plez 1998) from carbon-enhanced model atmospheres with the $C$,

\footnotetext{
3 However, given the strong $\mathrm{CH}$ band at $437.0 \mathrm{~nm}$ present in $\mathrm{HE} 0017+0055$, it is clear that the star must be considered as a $\mathrm{CH}$ star (see Fig. 1e of Barnbaum et al. 1996). The atmospheric parameters of $\mathrm{CH}$ and $\mathrm{R}$ stars are very similar at any rate.
}

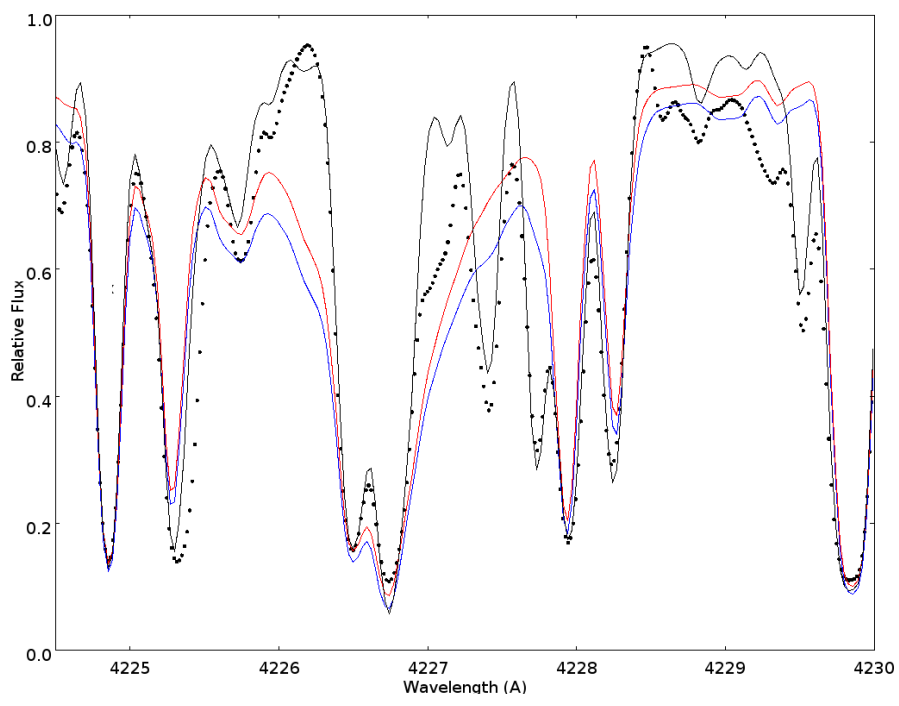

Fig. 4. Effect of gravity on the CaI $\lambda 422.67 \mathrm{~nm}$ line (black curve: $\log g=1$ and $[\mathrm{Fe} / \mathrm{H}]=-2.4$ dex; blue curve: $\log g=5$ and $[\mathrm{Fe} / \mathrm{H}]=$ -2.4 dex; red curve: $\log g=5$ and $[\mathrm{Fe} / \mathrm{H}]=-3.5 \mathrm{dex}$ ). Except otherwise stated, the atmospheric parameters used to generate the synthetic spectra are those listed in Table 4.

$\mathrm{N}$, and $\mathrm{O}$ abundances iteratively adapted to the values found in HE 0017+0055. The model atmospheres were computed with the opacity sampling technique usual for the MARCS models (Gustafsson et al. 2008), and using opacity tables with variable $\mathrm{C}, \mathrm{N}$, and $\mathrm{O}$ abundances.

The wings of the Balmer lines were fitted, as well as those of strong lines like Ca I $\lambda 422.67 \mathrm{~nm}$ (Fig. 4). Although the gravity and $s$-process overall abundances are entangled ( $s$-process elements often involve lines from the singly ionised species, which are generally strengthened in low-density atmospheres), a solution with $[\mathrm{s} / \mathrm{Fe}]=+2.0 \mathrm{dex}^{4}$ (or even more, except for the first $s$-process peak element $\mathrm{Y}$ ) and $\log g=1$ represents a good overall match to the spectrum, as shown in Fig. 5 in a region containing many lines from singly ionised, $s$-process elements. We stress that the shape of the ${ }^{12} \mathrm{C}^{13} \mathrm{C}$ and ${ }^{13} \mathrm{C}^{13} \mathrm{C}$ bandheads around $474.0 \mathrm{~nm}$ are sensitive to gravity (see Fig. 6), and it is this criterion that led us to adopt $\log g=1$.

HE $0017+0055$ cannot be a low-metallicity carbon dwarf, since even at this low metallicity, the wings of the more intense

4 This means that all heavy elements with the $s$ process contributing more than $50 \%$ of their solar system abundance were assigned an overabundance of 2.0 dex in the spectral synthesis computation. 


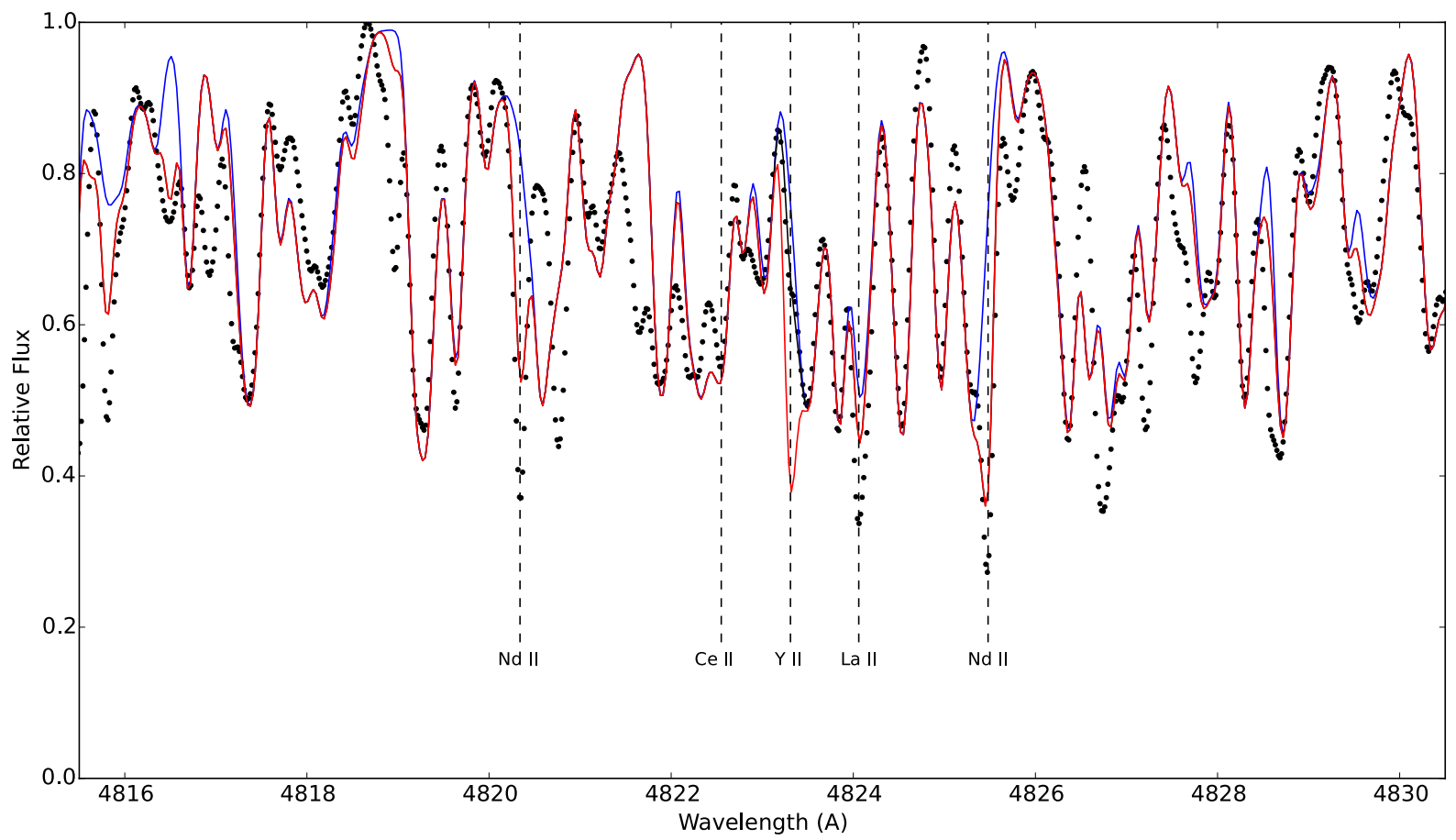

Fig. 5. Effect of a general increase by 2.0 dex of $s$-process elements (red line), as compared to their solar abundances (blue line). Compare with Fig. 5 of Reyniers et al. (2007). The Y II line around $4823.3 \AA$ is not as enhanced as the other $s$-process lines (red line). A better match to the $\mathrm{Y}$ abundance is obtained with $[\mathrm{Y} / \mathrm{Fe}]=0.5 \mathrm{dex}$ (black line). Except otherwise stated, the atmospheric parameters used to generate the synthetic spectra are those listed in Table 4.

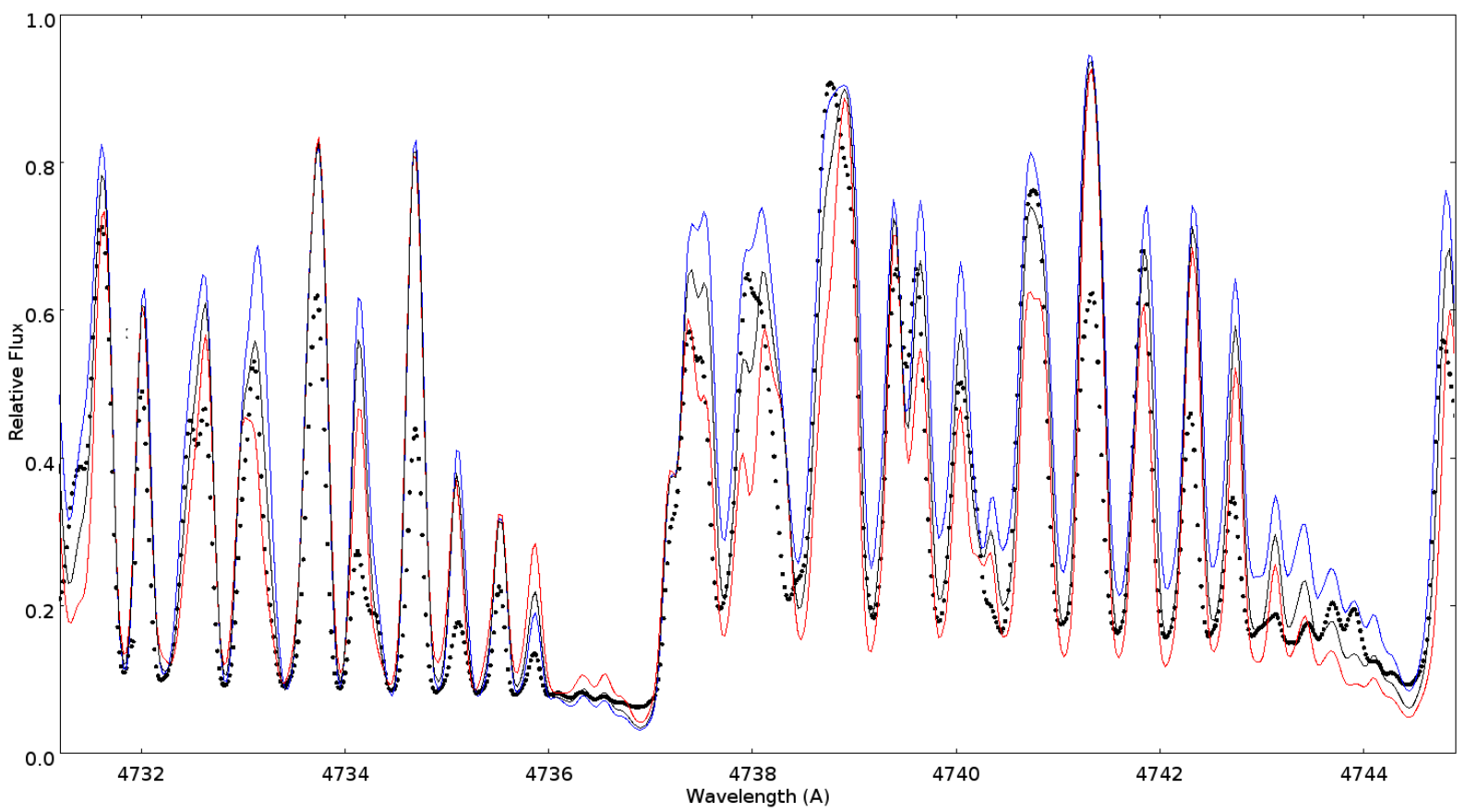

Fig. 6. ${ }^{12} \mathrm{C}^{13} \mathrm{C}$ and ${ }^{13} \mathrm{C}^{13} \mathrm{C}$ band heads around $474.0 \mathrm{~nm}$ (with $\log \epsilon(\mathrm{C})=8.2$ and $\log \epsilon(\mathrm{N})=7.9$ ) for ${ }^{12} \mathrm{C} /{ }^{13} \mathrm{C}=2$ (red curve), 4 (black curve), and 7 (blue curve). Except otherwise stated, the atmospheric parameters used to generate the synthetic spectra are those listed in Table 4.

lines would be too strong. Figure 4 reveals the impact of gravity on the Ca I $\lambda 422.67 \mathrm{~nm}$ line. At higher gravities, the wings of the line become stronger, and the metallicity has to be decreased (to $[\mathrm{Fe} / \mathrm{H}]=-3.5$ ), but then other (metallic) lines start becoming really weak and make this solution unacceptable.

Especially noteworthy is the fact that HE 0017+0055 combines features from both $\mathrm{J}$ stars (a low ${ }^{12} \mathrm{C} /{ }^{13} \mathrm{C}$ ratio, as inferred from the ${ }^{12} \mathrm{C}^{13} \mathrm{C}$ and ${ }^{13} \mathrm{C}^{13} \mathrm{C}$ bandheads around $474.0 \mathrm{~nm}$; see Fig. 6) and $\mathrm{CH}$ stars (large $s$-process overabundances, and strong $\mathrm{CH}$ bands in the region $430-440 \mathrm{~nm}$, caused by a large $\mathrm{C}$ overabundance and a very low metallicity $[\mathrm{Fe} / \mathrm{H}]=-2.4)$. The high $\mathrm{N}$ abundance, as derived from the $\mathrm{CN}$ band longwards of $570 \mathrm{~nm}$ or $800 \mathrm{~nm}$ (Fig. 7), is also remarkable. This $\mathrm{N}$ abundance is consistent with the low ${ }^{12} \mathrm{C} /{ }^{13} \mathrm{C}$ ratio $(\sim 4)$ 
Table 4. Adopted atmospheric parameters of HE $0017+0055$ ( $\xi_{\mathrm{t}}$ is the microturbulence).

\begin{tabular}{|c|c|c|c|c|c|c|c|c|c|c|c|}
\hline \multirow{2}{*}{$\begin{array}{l}T_{\text {eff }} \\
(\mathrm{K})\end{array}$} & \multirow[t]{2}{*}{$\log g$} & \multirow[t]{2}{*}[\mathrm{Fe}/\mathrm{H}]{} & \multicolumn{3}{|c|}{$\log \epsilon$} & \multicolumn{3}{|c|}{$[\mathrm{X} / \mathrm{H}]$} & \multirow[t]{2}{*}{${ }^{12} \mathrm{C} /{ }^{13} \mathrm{C}$} & \multirow[t]{2}{*}[\mathrm{s}/\mathrm{Fe}]{} & \multirow{2}{*}{$\begin{array}{c}\xi_{\mathrm{t}} \\
\left(\mathrm{km} \mathrm{s}^{-1}\right)\end{array}$} \\
\hline & & & $\mathrm{C}$ & $\mathrm{N}$ & $\mathrm{O}$ & $\mathrm{C}$ & $\mathrm{N}$ & $\mathrm{O}$ & & & \\
\hline $4250 \pm 100$ & $1 \pm 1$ & -2.4 & $8.2 \pm 0.1$ & $7.9 \pm 0.1$ & $7.1^{a}$ & -0.23 & 0.07 & $-1.6^{a}$ & $4 \pm 1$ & $2.0 \pm 0.2$ & 2 \\
\hline
\end{tabular}

Notes. ${ }^{a}$ Oxygen abundance imposed from $[\mathrm{O} / \mathrm{Fe}]=0.8$ (see Fig. 18 of Masseron et al. 2010).

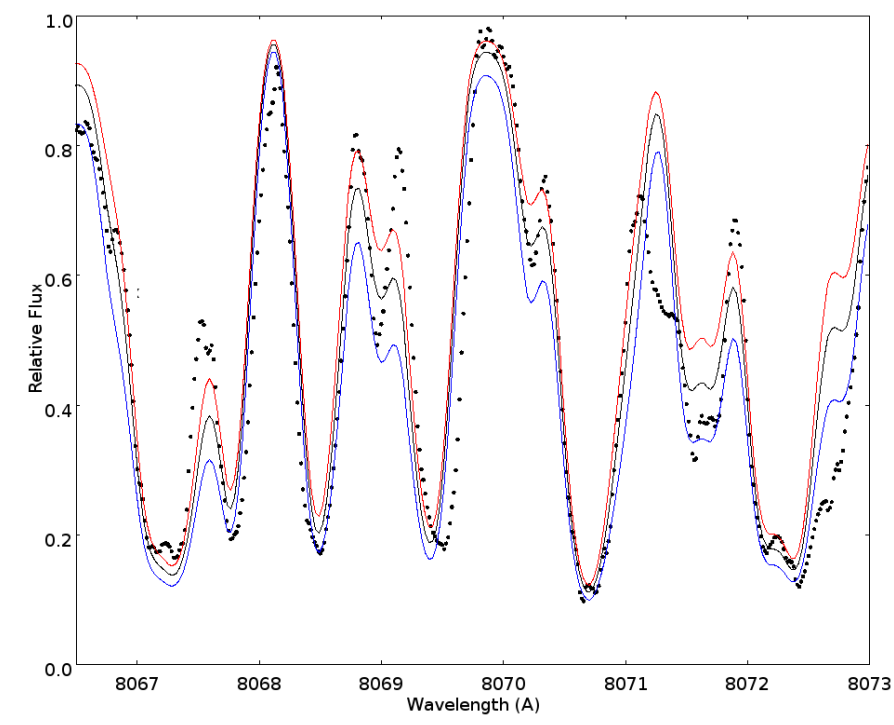

Fig. 7. Intensities of lines from the $\mathrm{CN}$ band around $\lambda 8070 \AA$, showing the impact of the $\mathrm{N}$ abundance, with $\log \epsilon(\mathrm{C})$ fixed at 8.2: $\log \epsilon(\mathrm{N})=$ 7.7 (red curve), $\log \epsilon(\mathrm{N})=7.9$ (black curve), $\log \epsilon(\mathrm{N})=8.2$ (blue curve). Except otherwise stated, the atmospheric parameters used to generate the synthetic spectra are those listed in Table 4.

since both features are signatures of $\mathrm{CN}$ processing, as may occur along the RGB, with extra mixing bringing these products to the stellar surface (Placco et al. 2014). The $\mathrm{N}$ overabundance $([\mathrm{N} / \mathrm{H}]=0.07,[\mathrm{C}+\mathrm{N} / \mathrm{H}]=-0.15$, and $[\mathrm{C} / \mathrm{N}]=-0.30)$ is in fact typical of CEMP-rs stars (we show in Sect. 4 that HE 0017+0055 is indeed a CEMP-rs star), as is shown in Fig. 17 of Masseron et al. (2010). With the adopted atmospheric parameters, there seems to be no way of avoiding a strong $\mathrm{N}$ overabundance if the strengths of the $\mathrm{CN}$ lines are to be reproduced. The carbon abundance of $\mathrm{HE} 0017+0055([\mathrm{C} / \mathrm{H}]=-0.23)$ seems reasonable among CEMP-rs stars as well (Fig. 14 from Masseron et al. 2010).

In conclusion, HE 0017+0055 must thus be considered another member of the CEMP class, albeit one of the coolest and most luminous member of the class, but not as extreme as CS 30322-023, the latter having atmospheric parameters $T_{\text {eff }}=$ $4100 \mathrm{~K}, \log g \leq-0.3$, and $[\mathrm{Fe} / \mathrm{H}]=-3$ (Masseron et al. 2006). The atmospheric parameters of HE $0017+0055$ are used in Sect. 4 to derive abundances for $s$-process elements. The evolutionary status of HE 0017+0055 is discussed next (Sect. 5).

\section{Heavy-element abundances}

A detailed analysis of abundances for some key elements (some typical of the $s$ process, others typical of the $r$ process) has been performed using the parameters from Table 4, using the Keck/Hires spectrum obtained on October 7, 2008 and reduced with the standard Makee pipeline. The line list is given in Table A.1, avoiding spectral regions with the unaccounted $\mathrm{SiC}_{2}$ Merrill-Sanford bands. Oscillator strengths are from the VALD database (Kupka et al. 2011). The corresponding abundances are
Table 5. Abundances (in the scale $\log \epsilon(\mathrm{H})=12$ ) of Fe and heavy elements, the line-to-line standard deviation is between parenthesis.

\begin{tabular}{lcrrr}
\hline \hline $\mathrm{X}$ & $\log \epsilon(X)$ & {$[X / \mathrm{H}]$} & {$[X / \mathrm{Fe}]$} & $N$ \\
\hline Fe & $5.02(0.07)$ & -2.4 & - & 6 \\
Y II & $0.33(0.03)$ & -1.9 & 0.5 & 2 \\
Zr II & $1.73(0.04)$ & -0.9 & 1.6 & 4 \\
La II & $1.12(0.13)$ & 0.0 & 2.4 & 5 \\
Ce II & $1.22(0.06)$ & -0.5 & 2.0 & 3 \\
Nd II & $1.20(0.06)$ & -0.3 & 2.2 & 7 \\
Sm II & $0.43(0.03)$ & -0.6 & 1.9 & 2 \\
Eu II & $0.35(0.05)$ & -0.2 & 2.3 & 2 \\
Dy II & $-0.10(0.20)$ & -1.2 & 1.2 & 2 \\
Er II & $0.25(0.05)$ & -0.7 & 1.8 & 2 \\
\hline
\end{tabular}

Notes. $N$ is the number of lines used in the computation of the average abundance. Abundances have been normalised by the solar photospheric values from Asplund et al. (2009).

listed in Table 5. The carbon-star model used has $[\mathrm{Fe} / \mathrm{H}]=-2$, $T_{\text {eff }}=4250 \mathrm{~K}, \log g=1.00,[\alpha / \mathrm{Fe}]=+0.4, \xi_{\mathrm{t}}=2.0 \mathrm{~km} \mathrm{~s}^{-1}$, $\log \epsilon(\mathrm{C})=8.2, \log \epsilon(\mathrm{N})=7.4, \log \epsilon(\mathrm{O})=7.1,[\mathrm{~s} / \mathrm{Fe}]=+2.0$ dex. The synthetic spectrum was convolved with a macroturbulence velocity of $8.0 \mathrm{~km} \mathrm{~s}^{-1}$. An analysis of the sensitivity of the Fe, $\mathrm{Zr}$, and Eu abundances to the atmospheric parameters is presented in Table 6. The global uncertainty, adding up all individual uncertainties, is of the order of \pm 0.3 dex for the heavy elements $\mathrm{Zr}$ and $\mathrm{Eu}$. This is somewhat larger than usual, but is nevertheless of the same order as the uncertainties found by Zamora et al. (2009) in their analysis of R-type carbon stars, thus revealing the difficulties inherent in the analysis of carbon stars.

The correct $\mathrm{Ce}$ abundance was used when deriving the $\mathrm{Li}$ abundance, since the Ce II line at $670.8099 \mathrm{~nm}$ blends the Li I resonance doublet at 670.7706 and $670.791 \mathrm{~nm}$ (Reyniers et al. 2002). We could only set an upper limit on the Li abundance: $\log \epsilon(\mathrm{Li})<1.0$. This star is thus not Li rich.

As indicated in Table 5, the heavy-element abundance pattern in HE 0017+0055 looks typical of a CEMP-s star at first sight, with moderate overabundances for elements belonging to the first $s$-process peak (Y, Zr), and stronger overabundances for the second peak ( $\mathrm{La}, \mathrm{Ce}, \mathrm{Nd}$ ). However, the analysis of the $r$-process elements $\mathrm{Sm}, \mathrm{Eu}, \mathrm{Dy}$, and Er reveals that they are enriched as well, and therefore HE 0017+0055 is likely a CEMP-rs star. The defining criterion of a CEMP-rs star is based on the $[\mathrm{Ba} / \mathrm{Eu}]$ ratio being in the range $0.0-+0.5$ dex, whereas $[\mathrm{Ba} / \mathrm{Eu}]>0.5 \mathrm{dex}$ for CEMP-s stars (Beers \& Christlieb 2005, see also Figs. 1 and 2 of Masseron et al. 2010). Unfortunately, the $\mathrm{Ba}$ abundance could not be derived safely because the 493.407, 585.37, and $614.14 \mathrm{~nm}$ lines were either blended or had strong and badly fitted wings. Nevertheless, a lower limit on the $\mathrm{Ba}$ abundance could be set at $\log \epsilon(\mathrm{Ba})>1.6$ dex (using the 493.407 and $614.14 \mathrm{~nm}$ lines), corresponding to $[\mathrm{Ba} / \mathrm{H}]>-0.6$ and $[\mathrm{Ba} / \mathrm{Fe}]>$ 1.9. Combined with $[\mathrm{Eu} / \mathrm{Fe}]=2.3 \operatorname{dex}($ Table 5), these values confirm the CEMP-rs nature of HE $0017+0055$ (see also Fig. 1 of Masseron et al. 2010). In that respect, it is 
Table 6. Sensitivity of the derived abundances (in dex) on the atmospheric parameters.

\begin{tabular}{lllllll}
\hline \hline Element & $T_{\text {eff }}$ & $\log g$ & {$[\mathrm{Fe} / \mathrm{H}]$} & $\xi$ & $\begin{array}{l}\text { Macro. } \\
1 \mathrm{~km} \mathrm{~s}^{-1}\end{array}$ & $\begin{array}{l}\text { Total } \\
(\mathrm{dex})\end{array}$ \\
\hline $\mathrm{Fe}$ & \pm 0.09 & \pm 0.07 & - & \pm 0.12 & \pm 0.03 & \pm 0.18 \\
$\mathrm{Zr}$ & \pm 0.20 & \pm 0.11 & \pm 0.07 & \pm 0.08 & \pm 0.11 & \pm 0.29 \\
$\mathrm{Eu}$ & \pm 0.20 & \pm 0.15 & \pm 0.20 & \pm 0.10 & \pm 0.07 & \pm 0.34 \\
\hline
\end{tabular}

Notes. For non-symmetrical abundance variations, the largest value is listed. The final uncertainty on the abundance is computed by quadratically adding all the uncertainties, including the standard deviation from the line-to-line scatter.

noteworthy that the $[\mathrm{La} / \mathrm{Ce}]$ ratio $(0.5 \mathrm{dex})$ is much larger than the typical (negative) values predicted by the computations of Goriely \& Siess (2005) from the operation of the ${ }^{13} \mathrm{C}(\alpha, n){ }^{16} \mathrm{O}$ neutron source in low-mass AGB stars. The larger [La/Ce] values observed in HE $0017+0055$ as in all the other CEMP-rs stars are thus a strong indication that the ${ }^{13} \mathrm{C}(\alpha, n){ }^{16} \mathrm{O}$ neutron source does not operate in those stars, as suggested by Masseron et al. (2010). It is also meaningful that the [La/Ce] values observed in CEMP-rs stars are compatible with the values $0.2-0.4$ dex predicted from the operation of the ${ }^{22} \mathrm{Ne}(\alpha, \mathrm{n})^{25} \mathrm{Mg}$ neutron source in warm pulses, and after dilution in the AGB envelope (Goriely \& Siess 2005).

Unfortunately, the abundance of $\mathrm{Pb}$, falling in the third $s$-process peak and generally much enhanced in CEMP-rs stars, could not be easily derived, as the $\mathrm{Pb}$ blend is not cleanly reproduced.

We could only set an upper limit on the technetium abundance $(\log \epsilon(T \mathrm{c})<0.0)$, which hints at the absence of Tc, and thus confirming the extrinsic nature of HE $0017+0055$.

\section{The evolutionary status of HE $0017+0055$}

The star must have formed in the early stage of the Galaxy since it is of very low metallicity. According to Fig. 2 of Freeman \& Bland-Hawthorn (2002), stars with metallicities $[\mathrm{Fe} / \mathrm{H}]=$ -2.4 , such as HE $0017+0055$ formed about 13 Gyr ago. Since HE $0017+0055$ is located on the giant branch, it must have spent about 12 Gyr on the main sequence (and the remainder in postmain-sequence phases) and must therefore have had an initial mass of about $0.9 M_{\odot}$. The gravity and effective temperatures of HE 0017+0055 mentioned in Sect. 3 may be combined, along with the above mass estimate $\left(0.9 M_{\odot}\right)$, to derive the luminosity

$\log L / L_{\odot}=\log M / M_{\odot}+4 \log T_{\text {eff }} / T_{\text {eff } \odot}-\log g / g_{\odot}=2.86$,

corresponding to $L=724 L_{\odot}$, in agreement with the prediction of the STAREVOL C-rich model described below (and Table 3). According to the compilation of CEMP-star properties by Masseron et al. (2010), this value for the luminosity makes HE $0017+0055$ one of the brightest CEMP stars (their Fig. 12), just below CS 30322-023 (Fig. 8 and Masseron et al. 2006). The radius corresponding to HE $0017+0055$ gravity and mass is $50 R_{\odot}$. Only CS 30322-023, with $\log g=-0.3 \pm 0.3$ and $T_{\text {eff }}=$ 4100-4350 K (Masseron et al. 2006), has a higher luminosity among CEMP stars. Although CS 30322-023 most likely lies above the RGB tip (Fig. 8), and might therefore belong to the rare class of low-metallicity AGB stars, this is not required for HE 0017+0055.

Figure 8 presents evolutionary tracks of a $0.9 M_{\odot}$ star with a metallicity of $[\mathrm{Fe} / \mathrm{H}]=-2.4$, as $\mathrm{HE} 0017+0055$, computed

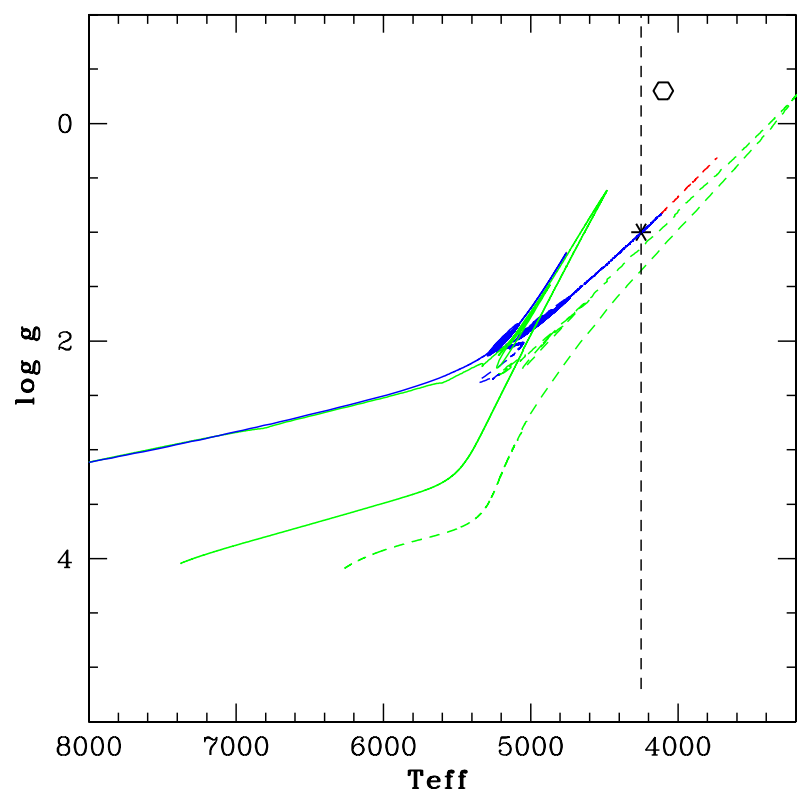

Fig. 8. Evolutionary track in the $\left(T_{\text {eff }}, \log g\right)$ diagram for a $0.9 M_{\odot}$ star of metallicity $[\mathrm{Fe} / \mathrm{H}]=-2.4$, computed with the STAREVOL code, with an atmosphere that is either oxygen-rich (solid track) or carbon-rich (dashed track, corresponding to $\log \epsilon(C)=8.2$ or $[\mathrm{C} / \mathrm{H}]=-0.23$ ). The oxygen-rich track is limited to the giant and horizontal branches (green and blue parts of the track, respectively), whereas the carbon-rich track includes the AGB (red part of the track) as well. The vertical dashed line corresponds to $T_{\text {eff }}=4250 \mathrm{~K}$. The location of HE $0017+0055$ as inferred from the parameters of Table 4 is indicated with the star symbol, whereas that of CS 30322-023 is indicated with the open circle.

with the STAREVOL code (Siess \& Arnould 2008). The exact location of the giant branch in the Hertzsprung-Russell diagram is sensitive to the opacity in the atmosphere, and hence to the carbon- or oxygen-rich nature of the star (Marigo 2002). For a star strongly enriched in carbon, as is HE 0017+0055, the evolutionary track is shifted towards lower temperatures, thus counteracting to some extent the bluewards shift due to the low metallicity. This effect appears crucial to derive atmospheric parameters consistent with those derived from the spectral analysis in Sect. 3.3, and especially to avoid the high luminosities that would yield large space motions.

The distance of HE 0017+0055 may then be estimated, adopting the same bolometric correction of 0.3 as that estimated by Masseron et al. (2006); for CS 30322-023, a distance of $4.8 \mathrm{kpc}$ is obtained (Table 3). At this kind of distance, the Tycho-2 proper motions (see Sect. 3.1) translate into large space velocities as listed in Table $3^{5}$. As discussed in Sect. 3.1, these space velocities are consistent with HE $0017+0055$ being a normal member of the Galactic halo.

An accurate trigonometric parallax for $\mathrm{HE} 0017+0055$ should become available from Gaia within a few years and should provide a conclusive answer to the discussion on the distance and evolutionary stage of this star.

\section{Conclusions}

The radial-velocity monitoring of $\mathrm{HE} 0017+0055$ revealed short-term variations superimposed on a long-term trend. The short-term variations are characterised by a period of $383 \mathrm{~d}$ and

\footnotetext{
5 A similar analysis is unfortunately not possible for CS 30322-023 for which no proper motion is available.
} 
a very small mass function of $(6.1 \pm 1.1) \times 10^{-6} M_{\odot}$. There are three possible explanations for these low-amplitude variations: (i) orbital variations caused by a WD companion in a very inclined orbit $\left(i \sim 2.3^{\circ}\right)$; (ii) orbital variations caused by a brown dwarf in a moderately inclined orbit $\left(12.4^{\circ} \leq i \leq 90^{\circ}\right)$; and (iii) velocity jitter reported by Carney et al. (2003) as typical in low-metallicity giants close to the RGB tip. Jorissen et al. (2016) report three more cases among CH/CEMP-s systems of small-amplitude velocity variations with periods close to $1 \mathrm{yr}$, very similar thus to that observed in HE 0017+0055. A definite conclusion as to which possibility is valid must await the results of an accurate photometric monitoring, since the variations are expected to be minute (a few hundredths of a magnitude).

An abundance study has shown that HE $0017+0055$ is not only enriched in $s$-process elements, but also in $r$-process elements (at levels of the order of 2 dex for most of them), and may thus be flagged as a CEMP-rs star. A mass-transfer scenario is generally invoked to account for the abundance peculiarities of CEMP-rs stars. In this scenario, the CEMP-rs star has been polluted by heavy-element-rich matter coming from a companion formerly on the thermally-pulsing AGB star (most probably a massive one; Masseron et al. 2010), which is now a WD. In case (i) above, the WD is in the inner orbit, and the outer orbit hosts an innocent bystander star; whereas in cases (ii) and (iii), the WD is in a long-period orbit. Although the outer orbit must have a period of several decades, this is still compatible with the mass-transfer scenario, since other $s$-process polluted systems with similarly long orbital periods are known (like HD 26; Jorissen et al. 2015).

Acknowledgements. This research has been funded by the Belgian Science Policy Office under contract BR/143/A2/STARLAB. S.v.E. and L.S. are FNRS research associates. The work of T.T.H. was supported by Sonderforschungsbereich SFB 881 "The Milky Way System" (subproject A4) of the German Research Foundation (DFG). J.A. and B.N. gratefully acknowledge financial support from the Danish Natural Science Research Council and the Carlsberg Foundation. Based on observations obtained with the HERMES spectrograph, supported by the Fund for Scientific Research of Flanders (FWO), the Research Council of K.U.Leuven, the Fonds National de la Recherche Scientifique (F.R.S.-FNRS), Belgium, the Royal Observatory of Belgium, the Observatoire de Genève, Switzerland, and the Thüringer Landessternwarte Tautenburg, Germany.

\section{References}

Abate, C., Pols, O. R., Izzard, R. G., Mohamed, S. S., \& de Mink, S. E. 2013, A\&A, 552, A26

Alonso, A., Arribas, S., \& Martinez-Roger, C. 1996, A\&A, 313, 873

Alvarez, R., \& Plez, B. 1998, A\&A, 330, 1109

Aoki, W., Beers, T. C., Christlieb, N., et al. 2007, ApJ, 655, 492

Asplund, M., Grevesse, N., Sauval, A. J., \& Scott, P. 2009, ARA\&A, 47, 481

Barbuy, B., Cayrel, R., Spite, M., et al. 1997, A\&A, 317, L63

Barbuy, B., Spite, M., Spite, F., et al. 2005, A\&A, 429, 1031

Barnbaum, C., Stone, R. P. S., \& Keenan, P. C. 1996, ApJS, 105, 419

Beers, T. C., \& Christlieb, N. 2005, ARA\&A, 43, 531

Beers, T. C., Flynn, C., Rossi, S., et al. 2007a, ApJS, 168, 128

Beers, T. C., Sivarani, T., Marsteller, B., et al. 2007b, AJ, 133, 1193
Bergeat, J., Knapik, A., \& Rutily, B. 2001, A\&A, 369, 178

Buchhave, L. A., Bakos, G. Á., Hartman, J. D., et al. 2010, ApJ, 720, 1118

Burgasser, A. J., Kirkpatrick, J. D., Burrows, A., et al. 2003, ApJ, 592, 1186

Burningham, B., Smith, L., Cardoso, C. V., et al. 2014, MNRAS, 440, 359

Carney, B. W., Laird, J. B., Latham, D. W., \& Aguilar, L. A. 1996, AJ, 112, 668

Carney, B. W., Latham, D. W., Stefanik, R. P., Laird, J. B., \& Morse, J. A. 2003, AJ, 125, 293

Carollo, D., Freeman, K., Beers, T. C., et al. 2014, ApJ, 788, 180

Christlieb, N., Green, P. J., Wisotzki, L., \& Reimers, D. 2001, A\&A, 375, 366

Cutri, R. M., Skrutskie, M. F., van Dyk, S., et al. 2003, 2MASS VizieR Online Data Catalog: II/246

Fabrycky, D., \& Tremaine, S. 2007, ApJ, 669, 1298

Freeman, K., \& Bland-Hawthorn, J., 2002, ARA\&A, 40, 487

Girardi, L., Bressan, A., Bertelli, G., \& Chiosi, C. 2000, A\&AS, 141, 371

Goriely, S., \& Siess, L. 2005, in From Lithium to Uranium: Elemental Tracers of Early Cosmic Evolution, eds. V. Hill, P. François, \& F. Primas, IAU Symp., 228,451

Gorlova, N., Van Winckel, H., Vos, J., et al. 2013, EAS Pub. Ser., 64, 163

Goswami, A. 2005, MNRAS, 359, 531

Gustafsson, B., Edvardsson, B., Eriksson, K., et al. 2008, A\&A, 486, 951

Hansen, T., Andersen, J., Nordström, B., Buchhave, L. A., \& Beers, T. C. 2011, ApJ, 743, L1

Hansen, T., Andersen, J., Nordström, B., et al. 2016, A\&A, in press, DOI: 10.1051/0004-6361/201527409

Hill, V., Barbuy, B., Spite, M., et al. 2000, A\&A, 353, 557

Høg, E., Fabricius, C., Makarov, V. V., et al. 2000, A\&A, 355, L27

Hummel, C. A., Benson, J. A., Hutter, D. J., et al. 2003, AJ, 125, 2630

Johnson, J. A., Herwig, F., Beers, T. C., \& Christlieb, N. 2007, ApJ, 658, 1203

Jorissen, A., Van Eck, S., Van Winckel, H., et al. 2016, A\&A, 586, A158

Kennedy, C. R., Sivarani, T., Beers, T. C., et al. 2011, AJ, 141, 102

Kiss, L. L., \& Bedding, T. R. 2003, MNRAS, 343, L79

Kjeldsen, H., \& Bedding, T. R. 1995, A\&A, 293, 87

Kupka, F., Dubernet, M.-L., \& VAMDC Collaboration 2011, Balt. Astron., 20, 503

Lucatello, S., Tsangarides, S., Beers, T. C., et al. 2005, ApJ, 625, 825

Marigo, P. 2002, A\&A, 387, 507

Marigo, P., Girardi, L., Chiosi, C., \& Wood, P. R. 2001, A\&A, 371, 152

Masseron, T., van Eck, S., Famaey, B., et al. 2006, A\&A, 455, 1059

Masseron, T., Johnson, J. A., Plez, B., et al. 2010, A\&A, 509, A93

McClure, R. D., \& Woodsworth, A. W. 1990, ApJ, 352, 709

Muterspaugh, M. W., Lane, B. F., Fekel, F. C., et al. 2008, AJ, 135, 766

O'Brien, D. P., McAlister, H. A., Raghavan, D., et al. 2011, ApJ, 728, 111

Pereira, C. B., Jilinski, E., Drake, N. A., et al. 2012, A\&A, 543, A58

Placco, V. M., Frebel, A., Beers, T. C., \& Stancliffe, R. J. 2014, ApJ, 797, 21

Pojmanski, G. 1997, Acta Astron., 47, 467

Raskin, G., van Winckel, H., Hensberge, H., et al. 2011, A\&A, 526, A69

Reyniers, M., Van Winckel, H., Biémont, E., \& Quinet, P. 2002, A\&A, 395, L35

Reyniers, M., Abia, C., van Winckel, H., et al. 2007, A\&A, 461, 641

Siess, L., \& Arnould, M. 2008, A\&A, 489, 395

Sivarani, T., Bonifacio, P., Molaro, P., et al. 2004, A\&A, 413, 1073

Smith, M. C., Ruchti, G. R., Helmi, A., et al. 2007, MNRAS, 379, 755

Stephenson, C. B. 1985, AJ, 90, 784

Stephenson, C. B. 1989, A general catalogue of cool carbon stars (Cleveland Publ. Warner \& Swasey Observatory)

Sterzik, M. F., \& Tokovinin, A. A. 2002, A\&A, 384, 1030

Udry, S., Mayor, M., \& Queloz, D. 1999, Precise Stellar Radial Velocities, IAU Colloq., 170, ASP Conf. Ser., 185, 367

Van Winckel, H., Jorissen, A., Gorlova, N., et al. 2010, Mem. Soc. Astron. It., 81,1022

Začs, L., Sperauskas, J., Grankina, A., et al. 2015, ApJ, 803, 17

Zamora, O., Abia, C., Plez, B., Domínguez, I., \& Cristallo, S. 2009, A\&A, 508, 909 


\section{Appendix A: Line list for the abundance analysis}

Table A.1. Lines used in the metallicity and $s$-process abundance analysis and the corresponding abundances (in the scale $\log \epsilon(\mathrm{H})=12$ ).

\begin{tabular}{|c|c|c|c|c|}
\hline & $\begin{array}{c}\lambda \\
(\AA)\end{array}$ & $\begin{array}{c}\chi_{\mathrm{exc}} \\
(\mathrm{eV})\end{array}$ & $\log g f$ & $\log \epsilon$ \\
\hline \multirow[t]{7}{*}{ Fe I } & 4466.551 & 2.831 & -0.600 & 4.9 \\
\hline & 4903.310 & 2.882 & -0.926 & 5.0 \\
\hline & 5195.472 & 4.220 & -0.086 & 5.1 \\
\hline & 6219.281 & 2.198 & -2.434 & 5.0 \\
\hline & 6839.830 & 2.559 & -3.350 & 5.0 \\
\hline & 7760.897 & 5.486 & -3.838 & 5.1 \\
\hline & & & & $5.02(0.07)$ \\
\hline \multirow[t]{3}{*}{ Y II } & 5200.406 & 0.992 & -0.570 & 0.35 \\
\hline & 5205.724 & 1.033 & -0.193 & 0.3 \\
\hline & & & & $0.325(0.025)$ \\
\hline \multirow[t]{5}{*}{$\mathrm{Zr}$ II } & 4457.413 & 1.184 & -1.220 & 1.7 \\
\hline & 4816.500 & 1.011 & -2.000 & 1.8 \\
\hline & 4831.327 & 1.208 & -1.720 & 1.7 \\
\hline & 4962.310 & 0.972 & -2.000 & 1.7 \\
\hline & & & & $1.73(0.04)$ \\
\hline \multirow[t]{23}{*}{ La II } & 4558.457 & 0.321 & -0.970 & 0.9 \\
\hline & 4808.996 & 0.235 & -1.40 & 1.3 \\
\hline & 4824.052 & 0.651 & -0.87 & 1.1 \\
\hline & 4920.965 & 0.126 & -2.261 & \\
\hline & 4920.965 & 0.126 & -2.407 & \\
\hline & 4920.966 & 0.126 & -2.065 & \\
\hline & 4920.966 & 0.126 & -2.078 & \\
\hline & 4920.966 & 0.126 & -2.738 & \\
\hline & 4920.968 & 0.126 & -1.831 & \\
\hline & 4920.968 & 0.126 & -1.956 & \\
\hline & 4920.968 & 0.126 & -2.629 & \\
\hline & 4920.971 & 0.126 & -1.646 & \\
\hline & 4920.971 & 0.126 & -1.895 & \\
\hline & 4920.971 & 0.126 & -2.650 & \\
\hline & 4920.975 & 0.126 & -1.490 & \\
\hline & 4920.975 & 0.126 & -1.891 & \\
\hline & 4920.975 & 0.126 & -2.760 & \\
\hline & 4920.979 & 0.126 & -1.354 & \\
\hline & 4920.979 & 0.126 & -1.957 & \\
\hline & 4920.979 & 0.126 & -2.972 & \\
\hline & 4920.985 & 0.126 & -1.233 & \\
\hline & 4920.985 & 0.126 & -2.162 & \\
\hline & 4920.985 & 0.126 & -3.375 & 1.2 \\
\hline
\end{tabular}

Notes. The bold values at the end of each element's list correspond to the average abundance and its corresponding standard dispersion. A vertical bar to the left of the wavelength values groups hyperfine structure and isotopic shifts for a given line.
Table A.1. continued.

\begin{tabular}{|c|c|c|c|c|}
\hline & $\begin{array}{c}\lambda \\
(\AA)\end{array}$ & $\begin{array}{l}\chi_{\text {exc }} \\
(\mathrm{eV}) \\
\end{array}$ & $\log g f$ & $\log \epsilon$ \\
\hline La II & $\begin{array}{l}4921.774 \\
4921.774 \\
4921.774 \\
4921.775 \\
4921.775 \\
4921.775 \\
4921.776 \\
4921.776 \\
4921.776 \\
4921.776 \\
4921.776 \\
4921.776 \\
4921.777 \\
4921.777 \\
4921.777 \\
4921.778 \\
4921.778 \\
4921.778 \\
4921.778 \\
4921.778 \\
4921.778\end{array}$ & $\begin{array}{l}0.244 \\
0.244 \\
0.244 \\
0.244 \\
0.244 \\
0.244 \\
0.244 \\
0.244 \\
0.244 \\
0.244 \\
0.244 \\
0.244 \\
0.244 \\
0.244 \\
0.244 \\
0.244 \\
0.244 \\
0.244 \\
0.244 \\
0.244 \\
0.244\end{array}$ & $\begin{array}{l}-1.139 \\
-2.220 \\
-3.601 \\
-1.233 \\
-2.005 \\
-3.207 \\
-1.334 \\
-1.445 \\
-1.915 \\
-1.927 \\
-2.923 \\
-3.010 \\
-1.566 \\
-1.955 \\
-2.939 \\
-1.700 \\
-1.848 \\
-2.006 \\
-2.053 \\
-2.258 \\
-3.123\end{array}$ & $\begin{array}{l}1.1 \\
\mathbf{1 . 1 2}(\mathbf{0 . 1 3})\end{array}$ \\
\hline Ce II & $\begin{array}{l}4835.674 \\
5187.458 \\
5191.633\end{array}$ & $\begin{array}{l}0.957 \\
1.212 \\
0.869\end{array}$ & $\begin{array}{l}-0.870 \\
0.150 \\
-0.560\end{array}$ & $\begin{array}{l}1.2 \\
1.15 \\
1.3 \\
\mathbf{1 . 2 2}(\mathbf{0 . 0 6})\end{array}$ \\
\hline Nd II & $\begin{array}{l}4594.447 \\
4820.339 \\
4825.478 \\
4859.026 \\
5228.420 \\
5249.576 \\
5276.869\end{array}$ & $\begin{array}{l}0.205 \\
0.205 \\
0.182 \\
0.321 \\
0.380 \\
0.976 \\
0.859\end{array}$ & $\begin{array}{l}-1.360 \\
-0.920 \\
-0.420 \\
-0.440 \\
-1.280 \\
0.200 \\
-0.440\end{array}$ & $\begin{array}{l}1.2 \\
1.28 \\
1.2 \\
1.1 \\
1.3 \\
1.2 \\
1.15 \\
\mathbf{1 . 2 0}(\mathbf{0 . 0 6})\end{array}$ \\
\hline Sm II & $\begin{array}{l}4777.840 \\
4815.800\end{array}$ & $\begin{array}{l}0.040 \\
0.185\end{array}$ & $\begin{array}{l}-1.420 \\
-0.820\end{array}$ & $\begin{array}{l}0.45 \\
0.4 \\
\mathbf{0 . 4 3}(\mathbf{0 . 0 3})\end{array}$ \\
\hline
\end{tabular}


Table A.1. continued.

\begin{tabular}{|c|c|c|c|c|}
\hline & $\begin{array}{c}\lambda \\
(\AA)\end{array}$ & $\begin{array}{l}\chi_{\text {exc }} \\
(\mathrm{eV})\end{array}$ & $\log g f$ & $\log \epsilon$ \\
\hline $\mathrm{Eu} \mathrm{II}$ & $\begin{array}{l}4522.486 \\
4522.498 \\
4522.538 \\
4522.549 \\
4522.561 \\
4522.581 \\
4522.590 \\
4522.600 \\
4522.615 \\
4522.621 \\
4522.630 \\
4522.640 \\
4522.644 \\
4522.650 \\
4522.657 \\
4522.661\end{array}$ & $\begin{array}{l}0.207 \\
0.207 \\
0.207 \\
0.207 \\
0.207 \\
0.207 \\
0.207 \\
0.207 \\
0.207 \\
0.207 \\
0.207 \\
0.207 \\
0.207 \\
0.207 \\
0.207 \\
0.207\end{array}$ & $\begin{array}{l}-2.159 \\
-1.266 \\
-1.984 \\
-1.474 \\
-2.159 \\
-1.962 \\
-1.711 \\
-1.984 \\
-2.038 \\
-1.980 \\
-1.962 \\
-2.247 \\
-2.256 \\
-2.038 \\
-2.344 \\
-2.247\end{array}$ & 0.3 \\
\hline & $\begin{array}{l}6437.637 \\
6437.639 \\
6437.635 \\
6437.637 \\
6437.637 \\
6437.635 \\
6437.635 \\
6437.633 \\
6437.633 \\
6437.630 \\
6437.623 \\
6437.627 \\
6437.620 \\
6437.606 \\
6437.617 \\
6437.603\end{array}$ & $\begin{array}{l}1.320 \\
1.320 \\
1.320 \\
1.320 \\
1.320 \\
1.320 \\
1.320 \\
1.320 \\
1.320 \\
1.320 \\
1.320 \\
1.320 \\
1.320 \\
1.320 \\
1.320 \\
1.320\end{array}$ & $\begin{array}{l}-1.428 \\
-2.206 \\
-2.206 \\
-1.377 \\
-2.010 \\
-2.010 \\
-1.287 \\
-1.956 \\
-1.956 \\
-1.181 \\
-1.998 \\
-1.998 \\
-1.070 \\
-2.191 \\
-2.191 \\
-0.960\end{array}$ & $\begin{array}{l}0.4 \\
\mathbf{0 . 3 5}(\mathbf{0 . 0 5})\end{array}$ \\
\hline Dy II & $\begin{array}{l}3984.688 \\
3996.689\end{array}$ & $\begin{array}{l}1.752 \\
0.590\end{array}$ & $\begin{array}{l}-0.328 \\
-0.260\end{array}$ & $\begin{array}{l}+0.1 \\
-0.3 \\
-\mathbf{0 . 1 0}(\mathbf{0 . 2 0})\end{array}$ \\
\hline Er II & $\begin{array}{l}3980.144 \\
4008.177\end{array}$ & $\begin{array}{l}0.886 \\
0.055\end{array}$ & $\begin{array}{l}-1.004 \\
-1.547\end{array}$ & $\begin{array}{l}0.2 \\
0.3 \\
\mathbf{0 . 2 5}(\mathbf{0 . 0 5})\end{array}$ \\
\hline
\end{tabular}

